\title{
Building Progressive Confidence: the Transition from Project to Operational Opening in the Case of a Major New International Airport Terminal
}

\author{
Vedran Zerjav', Andrew Davies ${ }^{1}$, Andrew Edkins ${ }^{1}$ and Philip Jones ${ }^{2}$ \\ ${ }^{1}$ Bartlett School of Construction and Project Management, University College London, UK \\ ${ }^{2}$ PJ Professional Management Solutions Ltd.
}

\begin{abstract}
The purpose of an infrastructure system is to deliver a variety of services to end users over long periods of time. One of the biggest challenges in the design and construction of new infrastructure facilities is the transition or handover from the project to operations. This is particularly the case for infrastructural assets that are complex in their operations such as energy generation plants, airports, ships and aircraft carriers, or hospitals. A variety of commissioning, testing, systems integration and operational readiness procedures have to be put in place to ensure a smooth handover to end users and operators. This paper presents an ongoing empirical study that investigates the challenge of delivering the operational outcomes of Heathrow Terminal 2 (T2), a major international airport hub terminal. The study consists of site observations, four preliminary and 15 in-depth interviews with highly knowledgeable informants in key positions concerning the delivery of the project and airport services to the end-users. Preliminary findings indicate that a specific form of high reliability focus which our participants called 'progressive confidence' is essential for the smooth transition from project to operations. The emergent findings suggest that this process comprises specific aspects of organisational learning, the notion of the "flip" between the project and operations and approaches for dealing with change. The findings suggest the importance of further research into the issues of operational readiness and transitioning towards the handover of complex infrastructure projects.
\end{abstract}

Keywords: Infrastructure Delivery Challenge, Airports, Reliability, Operational Readiness

\section{INTRODUCTION}

Large-scale infrastructure is planned, designed, built, and delivered through temporary project organisations involving a large coalition of stakeholders. These projects may be required to maintain and upgrade existing facilities or build entirely new infrastructure systems. One of the most critical points in the lifecycle of any large-scale infrastructure is the transition and handover of the project to its end users and operators. This involves a shift in organisational structure, skills and culture from a temporary project to a permanent operator. It is at this stage that the organisation moves from design, construction, systems integration, testing and commissioning to operation and the provision of services to end users.

As a result, owners of complex infrastructure projects must pay particular attention to the handover from the project to operations. The primary purpose of handover is to enable a smooth transition between construction and operations. Even though the role of operational handover is relatively well investigated in the in IT, defence, and aerospace industries, relatively little is known about key organisational routines and practices that lead to the successful transition from project to operational outcomes for infrastructure projects. This is surprising, given the key 
role that the handover stage plays in the lifecycle such projects. It is clear that disruptions or failures in handover can create severe reputational damage and loss of revenues for the infrastructure owner and operator.

To address this gap, the purpose of this paper is to present a study that investigates reliability as a key feature of infrastructure handover. This will be accomplished through an empirical study focusing on the various organisations involved with the project-operations delivery phase of a major international airport hub terminal.

\section{NORMAL ACCIDENTS AND HIGH RELIABILITY ORGANISING}

To investigate reliability aspects of the transition phase between the project and operations, we draw from organisation theory as a theoretical framework. The two most prominent strands of research that have tackled the organisational reliability issues are normal accidents theory ${ }^{1}$ (NAT) and high reliability theory ${ }^{2}$ (HRT). NAT argues that accidents are inevitable or normal in interactively-complex and tightly-coupled systems where localized incidents can quickly escalate and cause the entire system to fail. HRT, on the other hand, contends that there are certain organisations that manage to maintain a remarkably low occurrence of accidents in spite of the highly uncertain and changing conditions in which they operate. Examples of such organisations include nuclear power plants, aircraft carriers, submarines, and air traffic control systems. These organisations are referred to as high reliability organizations (HROs) $)^{3}$. Further research ${ }^{4}$ has shown that, to maintain their remarkable safety performance, HROs operate by implementing the principles of: (1) Preoccupation with failure, (2) Reluctance to simplify interpretations, (3) Sensitivity to operations (4) Commitment to resilience, and (5) Deference of expertise.

Our intention in this paper is to speak to the existing debates in the field of organisational reliability and address the identified challenges of the systematic view ${ }^{5}$ and the importance of the temporal dimension 6 . To this end, we employ an empirical research study that focuses on reliability in the project-operations transition stage of a large airport reconstruction project. After having elucidated the theoretical perspective and purpose of this study, we now turn to the research design and method. We also hope to contribute to the project studies and organisational literature by deepening our understanding of the often neglected transition to operations in the back-end of project life cycle.

\section{RESEARCH DESIGN AND DATA COLLECTION}

We selected the project to operations transition stage in the setting of an airport terminal because prior research suggested that this is a difficult and poorly understood activity ${ }^{7}$. As experience with some major international airports has shown, problems that occur during the transition to airport operations can rapidly change the public perception of an otherwise successful project. One of the notable examples of such a situation is the hugely disrupted handover of Heathrow Terminal 5 in 2008 due to the poor coordination between BAA, the airport owner, and British Airways, the eventual occupier of the new building ${ }^{8}$.

The empirical research is designed as case study ${ }^{9}$ of Heathrow Terminal 2 (T2), at the time of conducting the present study, an ongoing airport reconstruction project worth £2.5 billion (current price). Apart from its sheer scale, the complexity of the project was associated with creating a new terminal in a fully operational, extremely busy and

\footnotetext{
1 Perrow, C. (2011). Normal Accidents: Living with High Risk Technologies (Updated). Princeton University Press

2 Weick, K. E., and Sutcliffe, K. M. (2011). Managing the Unexpected: Resilient Performance in an Age of Uncertainty. Vol. 8, John Wiley \& Sons

3 La Porte, T. R. (1996). "High Reliability Organizations: Unlikely, Demanding and at Risk." Journal of contingencies and crisis management, 4(2), 60-71.

4 Weick, K. E., and Sutcliffe, K. M. (2011). Managing the Unexpected: Resilient Performance in an Age of Uncertainty. Vol. 8, John Wiley \& Sons.

5 Leveson, N., Dulac, N., Marais, K. and Carroll, J. (2009). "Moving Beyond Normal Accidents and High Reliability Organizations: A Systems Approach to Safety in Complex Systems." Organization Studies, 30(2-3), 227-249

6 Shrivastava, S., Sonpar, K. and Pazzaglia, F. (2009). "Normal Accident Theory Versus High Reliability Theory: A Resolution and Call for an Open Systems View of Accidents." Human Relations, 62(9), 1357-1390.

7 Davies, A., Gann, D. and Douglas, T. (2009). "Innovation in Megaprojects: Systems Integration at London Heathrow Terminal 5." California Management Review, 51(2).

8 Brady, T., and Davies, A. (2010). "From Hero to Hubris-Reconsidering the Project Management of Heathrow's Terminal 5." International Journal of Project Management, 28(2), 151-157.

9 Yin, R. K. (2011). Applications of Case Study Research. Sage
} 
congested airport, where operations had to be maintained despite the ongoing works. Motivated by lessons learned from various airport openings worldwide, the transition from the project to operations played a major role in the project. Reliability and safety were expressly a key part of the organisational culture in this project which makes it a particularly relevant research setting to study aspects of reliability in the delivery of infrastructure operations to the end users. Moreover, the airport opening was publicly perceived as successful and various public media made recurrent references that attribute the successful opening to the carefully planned transition from construction to becoming operational. A number of organisations had to work interdependently during the handover including a new Operational Readiness (OR) team established to achieve a smooth opening.

This study aimed to answer the following research question: What the organisation responsible for creating the new airport terminal did to successfully manage the transition from project mode to operations mode? To address this research question, we designed a study that focuses on understanding the role, practices and interactions of the various organisations involved in delivering the new terminal building. The exploratory phase of the research comprised a one-day observational visit of the project, whereby we conducted preliminary conceptual interviews ${ }^{10}$ with three highly-knowledgeable informants. The outcome of this preliminary phase was the formulation of the above research qu estion and preliminary validation of the relevance of a study focusing on the transition towards operational delivery.

To explore the conditions required to ensure a reliable handover, we approached a number of key informants in Heathrow Airport Limited, the project client organisation, to interview them about key aspects of safety and reliability as part of their organisational culture in delivering this airport project. The key informants were selected from different levels of the organisation such as (1) leadership, (2) organisational units, and (3) technical systems teams (e.g., buildings, and information and communication systems).

\section{EMERGENT FINDINGS: BUILDING OF PROGRESSIVE CONFIDENCE}

Preliminary findings from the interviews and secondary project data indicate that reliability was a key aspect of the organisational culture both in the transition towards operational delivery as well as at the point of the project handover. Overall, emergent findings suggest a narrative in which the handover reliability can be conceived as a process of building progressive confidence as the project moves towards full operations. This progressive confidence is characterised by establishing routines, tests and guarantees to ensure a predictable transition process, whilst being constantly vigilant to the possibility of potential disruptions. Whilst the emphasis in progressive confidence building is on providing stability, there was an ever present recognition that the organisation had be able to quickly and effectively solve unexpected problems and events that might hamper the smooth transition to operations. We continue with summarising several streams of findings that are emerging from the ongoing data analysis.

\section{Organisational learning}

The informants made frequent references to lessons learned from a previous project that was delivered within the client organisation. The experience on this project was the basis for designing the handover effort of the project. This previous project experience was the motivation to take a strategic focus on the delivery of services and prepare for the operations six months ahead of the go-live date and with the specific strategic focus on passenger experience. Similarly, on the basis of experiences from openings of other international airports, the overarching strategy for the operations was to implement a "soft" opening with sufficient buffers in the airport capacity to accommodate any unforeseen events and, as a result, to a achieve a formal opening that was a "non-event" in journalistic headlines terms. The period subsequent to the opening was, moreover, planned as a gradual ramp-up period of 4 months where the airport's operations would be scaled up to gradually reach their full operating capacity allowing for sufficient time to adapt the processes to the new setting. One of the results of the strong sense for organisational learning was to plan a specific moment in the project timeline when the organisational ownership of the project would be handed over from the construction and development team to the operational readiness team. This important milestone in the project was, in the data, referred to as the flip that is further described in more detail.

10 Spradley, J. P. (1979). The Ethnographic Interview. Holt, Rinehart and Winston New York. 


\section{The "flip" between project and operations}

The informants indicated that a particular date in the project timeline marked a significant shift of the emphasis from the construction project to the transitional project. This so called flip was referred to on numerous occasions by the informants in the context of events that occurred before or subsequent to it. While before the flip the focus was on system testing, after the flip the construction site was being used primarily for the purpose of staged trials. As opposed to technical testing of devices, the trials involved people and were organised in a succession going from physical units to entire areas in a progressively complex effort. The project involved 192 trials with a familiarisation program involving 3000 people in the final trials that also included a dummy live flight before the opening.

The gradual progression in the trials was organised in a way similar to the "soft" opening and gradual ramp-up plan for the airport operations. The rationale behind it was very well aligned with the basic principles of high-reliability organisations. Systems were established to ensure the performance of different aspects of user and operator interactions in the way that each subsequent trial was designed with an increased complexity and scale of system interactions. In such a way, the organisations involved in the transition towards the handover were dealing with high levels of uncertainty such as the numerous change requests that were generated as the project proceeded. This aspect is further elaborated in the following subsection.

\section{Dealing with change}

It was clear from the outset of the project that a combination of the complexity of the building and the rapidly changing aviation industry were likely to have an impact on the project as it unfolded with the requirement for changes. It is not surprising that this assumption proved to be true. Changes in scope, specification, and sequencing were a pervasive feature of the entire project, ranging from business changes of the airport tenant airlines, across policy-level changes in border control processes, towards the technological innovation of common check-in areas. As many of the changes were being implemented in the development and construction processes, they were particularly emphasised by the informants to have had a substantial impact on the trials and transition processes. As a result, the trials were often reporting difficulties as the changes were propagating throughout the airport project propelled by technical, business, social, as well as policy-level interdependencies that emerged but were unidentifiable from the outset of the project. One instance that describes the enactment of a chain of changes is the situation when an airline company expected to be a key tenant was taken over and, as a result, moved to another terminal in the airport. This caused a substantial reorganisation of the terminal building layout and processes because these were initially being developed to match the business model and processes of that particular airline company. As a consequence, the board of the client organisation negotiated a solution in which a major alliance (consisting of 23 international airlines) was chosen as the client of the airport development. This changed the project significantly because the various airlines did not necessarily share the same processes. As a result, a number of changes were instigated in the project at levels of technical support systems, layout of different parts of the facility, and design of the processes and passenger flows. This had a knock-on effect for the airlines themselves as not all of them had the processes in place to accommodate these changes. This awareness about the inevitability of change was ingrained in project practices and organisational culture. This translated into the level of organisational and business structure where the project used two separate contracts. Whereas one contract was specifying the requirements and defining the deliverables, a completely separate contract was in place to deal with all the incoming changes on the project. Summarising the emergent findings of the study, it can be said that the awareness of these changes and uncertainty led to a particular mind-set, characteristic for organisations that need to reliably operate in high-risk environments. We next discuss these findings in more detail.

\section{DISCUSSION AND CONCLUSIONS}

Practices of high-reliable organisations include preoccupation with failure, reluctance to simplify, sensitivity to operations, commitment to resilience, and deference to expertise. Different interpretations and examples that can be reduced to these particular features are pervasive in the interview accounts. Drawing from these inferences, we refer to the various reliability practices as the progressive building of confidence. This term aptly summarises the emergent findings from the ongoing analysis of the case study data. The notion of progressive confidence teases out the emergent aspects of interdependency between project and operations that are normally not taken into account in discussions around the delivery of infrastructure assets and services. The emergent findings are summarised in the figure below (Fig 1). 
This figure shows the relation between the activities of construction and operations. As opposed to the traditional project management body of knowledge that assumes projects entail discrete phases, our findings suggest that focusing on the discontinuities between these phases, in particular between construction and operations provides valuable insights into understanding the delivery of infrastructure services. Along the lines of this rationale, the handover of the project to the operators can be understood as a transitional phase that was labelled Operational Readiness in the case project. This transition can also be understood as a sub-project in its own right as:

- It is a temporary organisation with a specific purpose (i.e. to ensure a seamless delivery of infrastructure functionality to the users and operators),

- It is delivered in a limited timeframe (i.e. before the opening date)

- It requires substantial resources given its large scope (i.e. including technical tests and trials)

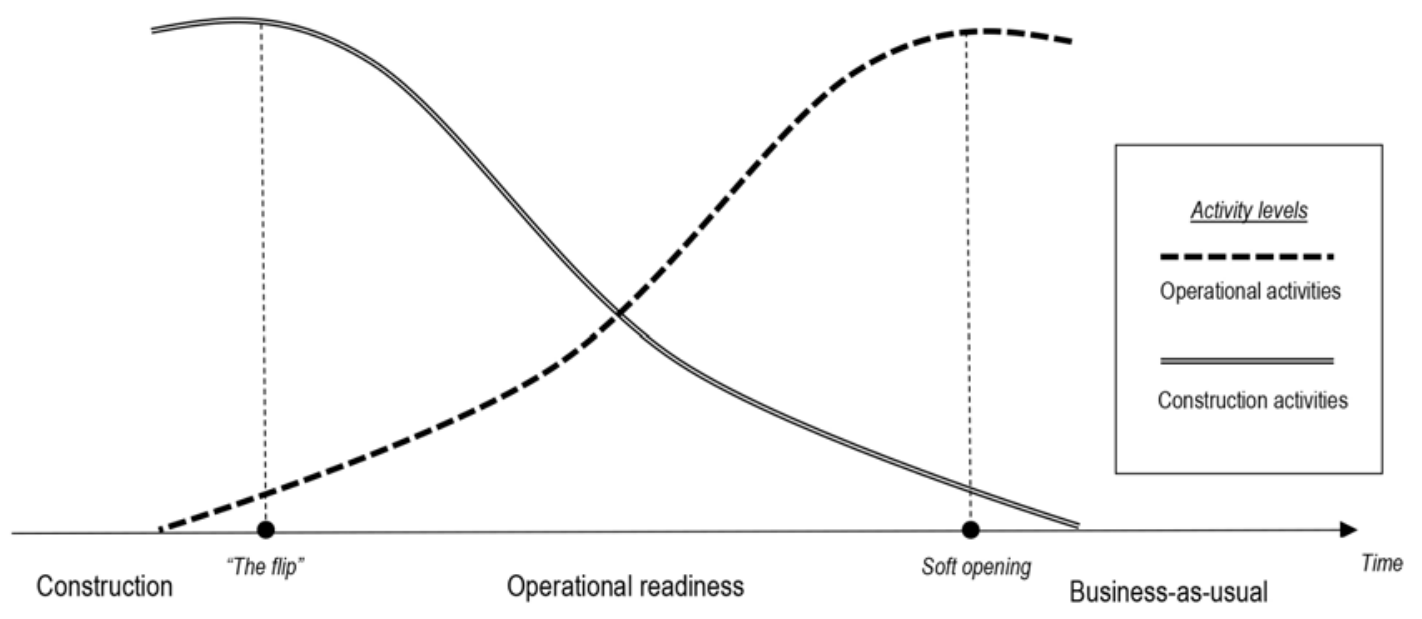

\section{Figure 1 - The transition between project and operations as the process of} "progressive building of confidence"

This transitional project is bounded by the construction activities on the one end and business-as-usual operations on the other end. In the T2 case study, it was characterised by a significant overlap between streams of construction and operation work. To be more specific, the operational activities were developed and delivered with a progressively increasing intensity, escalating from the construction phase to full capacity operations. At the same time, construction activities gradually decreased until the point the facility reaches full operational capacity. This transformation, understood as an interplay between construction and operational activities is, we argue, the distinguishing feature of a successful operational delivery.

The main practical contribution arising from this ongoing study is in the importance of the ex-ante strategy for the transition from a construction project towards a project to successfully deliver a fully functional operated environment. Although largely ignored by existing PM bodies of knowledge, we found that the broad awareness about the importance of achieving operational delivery in line with high reliability principles, was a key feature that pervaded both the interview accounts as well as secondary project documentation. This state of mind resulted in what this paper labels as the progressive building of confidence, an area that, we believe, deserves a lot more attention both from the perspective of practices as well as academic research.

\section{ACKNOWLEDGMENTS}

The authors would like to express their gratitude to the informants of the study whose details will not be disclosed. In particular, we would like to thank Sally Blackwell for sponsoring this research on behalf of Heathrow Airport Limited. Without her support, this study would have been impossible. Finally, ideas incorporated into this article are developed during research funded by the EPSRC and ESRC through the International Centre for Infrastructure Futures, which was also used to assist in providing the staff resource required to conduct the research. 